\title{
Role of Peroxisome Proliferator Activated Receptor-Gamma in Bacillus Calmette-Guérin Bladder Cancer Therapy
}

\author{
Yanina Langle, ${ }^{*}$ Catalina Lodillinsky, $†$ Denise Belgorosky,* Eduardo Omar Sandes \\ and Ana María Eiján*,‡
}

From the Área Investigación, Instituto de Oncología Ángel H. Roffo, University of Buenos Aires, Buenos Aires, Argentina

\begin{abstract}
Abbreviations and Acronyms

$15-d-P G J 2=15-$ deoxy- $\Delta 12-14$ prostaglandin J-2

$\alpha$-SMA $=\alpha$-smooth muscle actin

$\mathrm{BADGE}=$ bisphenol A diglycidyl

ether

$\mathrm{BC}=$ bladder cancer

$\mathrm{BCG}=$ bacillus Calmette-Guérin

$\mathrm{MAC}=$ macrophage

$\mathrm{MMP}=$ matrix metalloproteinase

$\mathrm{NF}-\kappa \mathrm{B}=$ nuclear factor $-\kappa \mathrm{B}$

$\mathrm{NMI}=$ nonmuscle invasive

PPAR $=$ peroxisome proliferator

activated receptor

PPRE $=$ PPARg responsive

element

RAW $=$ RAW264.7 cell

$\mathrm{RO}=$ rosiglitazone
\end{abstract}

Submitted for publication March 19, 2012

Study received approval from the Institutional Review Board CICUAL, Facultad de Medicina, Universidad de Buenos Aires, Argentina.

Supported by UBACYT M017 and PIP Consejo Nacional de Investigaciones Científicas y Técnicas 0193 .

* Financial interest and/or other relationship with the Consejo Nacional de Investigaciones Científicas y Técnicas.

† Current address: Institut Curie, UMR144, 26, rue d'Ulm, Paris 75248, Cedex 05, France.

‡ Correspondence: Área Investigación, Instituto de Oncología Ángel H. Roffo, Av. San Martín 5481, C 1417 DTB, Buenos Aires, Argentina (telephone and FAX: 54-11-4580-2811; e-mail: anamariaeijan@gmail.com.ar).

Purpose: We evaluated the effects of combined PPARg agonist with bacillus Calmette-Guérin in bladder cancer growth in vitro and in vivo, focusing on the tissue remodeling mechanisms induced by bacillus Calmette-Guérin.

Materials and Methods: PPARs are a superfamily of nuclear receptors that are transcription factors activated by ligands. Activation of PPARg, the $\gamma$ subtype, causes proliferation inhibition or differentiation of tumor cells. Previously, we reported that the inhibition of murine bladder tumor growth induced by bacillus Calmette-Guérin, which is the standard treatment for patients with nonmuscle invasive, high grade bladder cancer, increased PPARg expression in vitro and in vivo. In vitro the cell growth inhibition induced by bacillus Calmette-Guérin was enhanced by the PPARg agonist 15-d-PGJ2, raising the possibility that PPARg activation may be a therapeutic modality for this disease.

Results: In MB49 cells bacillus Calmette-Guérin and 15-d-PGJ2 induced PPARg expression, nuclear translocation and transcriptional activity. In vivo bacillus Calmette-Guérin reduced tumor size, an effect that was partially reversed when bacillus Calmette-Guérin was combined with the PPARg agonist rosiglitazone. The same result was found when we analyzed the effect of the PPARg antagonist BADGE (Fluka Chemical, Buchs, Switzerland) combined with bacillus CalmetteGuérin. Analysis of the activation of macrophages and fibroblasts demonstrated that rosiglitazone inhibited the tissue remodeling mechanisms induced by bacillus Calmette-Guérin.

Conclusions: Results suggest that PPARg is involved in the antitumor action of bacillus Calmette-Guérin. However, exogenous PPARg agonists would not be a favorable therapeutic modality because they can inhibit the tissue remodeling needed for an overall satisfactory bacillus Calmette-Guérin response.

Key Words: urinary bladder, urinary bladder neoplasms, PPAR gamma, BCG vaccine, regeneration

BLADDER cancer is the second most common tumor of the male urogenital tract and an important worldwide cause of death. ${ }^{1,2}$ The most common histological type is transitional cell carcinoma, representing more than $90 \%$ of cases. ${ }^{3}$ According to WHO invasion status, $\mathrm{BC}$ is classified as NMI and muscle invasive, the latter when it reaches the detrusor muscle. According to histological grade, $\mathrm{BC}$ can be a papillary urothelial neoplasm of low malignant potential, or low or high grade carcinoma. ${ }^{4}$

BCG is the standard therapy to prevent high grade NMI BC recurrence and progression. BCG triggers an immune response, ${ }^{5,6}$ induces in 
vitro apoptosis of $\mathrm{BC}$ cells ${ }^{7}$ and inhibits in vivo murine tumor growth. It induces stromal remodeling, including the differentiation of fibroblasts to myofibroblasts, collagen fiber deposits ${ }^{8}$ and MAC activation. ${ }^{9}$

PPARs are a superfamily of nuclear receptors that are transcription factors activated by ligands. ${ }^{10}$ The most active natural agonist of PPARg is 15-dPGJ2 ${ }^{11}$ Antidiabetic thiazolidinedione compounds, such as RO, are synthetic PPARg ligands. ${ }^{12}$ PPARg activation causes the apoptosis, growth inhibition or differentiation of BC cells. ${ }^{13,14}$ In addition, RO induces cell cycle arrest in BC cell lines. ${ }^{15}$ PPARg is expressed in normal urothelium and its expression in $\mathrm{BC}$ is associated with lower recurrence. ${ }^{16,17}$

Previously, we noted that BCG induced PPARg expression in murine $\mathrm{BC}$ cells in vitro and in vivo. ${ }^{18}$ In vitro 15-d-PGJ2 enhanced the MB49 BC cell line death induced by BCG. Based on these results, the combination of a PPARg agonist and BCG immunotherapy could be a potential tool for the treatment of NMI BC. ${ }^{19}$ In the current study we evaluated this hypothesis in vitro and in vivo using a MB49 BC model with a special focus on the tissue remodeling mechanisms induced by BCG.

\section{MATERIALS AND METHODS}

\section{Cell Culture and BCG}

The NIH-3T3 fibroblast cell line and MAC RAWs (ATCC ${ }^{\text {TM}}$ ) were maintained in Dulbecco's modified Eagle's medium (31600-026, Gibcoß). MB49 cells were cultured in RPMI 1640 (31800-014, Gibco). All culture medium was supplemented with $2 \mathrm{mM}$ L-glutamine, $80 \mu \mathrm{g} / \mathrm{ml}$ gentamicin and 5\% to $10 \%$ fetal bovine serum in a humidified atmosphere with $5 \% \mathrm{CO}_{2}$. We used living organisms $(3 \times$ $10^{6} \mathrm{cfu} / \mathrm{mg} / \mathrm{ml}$ ) of an attenuated strain of Mycobacterium tuberculosis (Pasteur 1172 P2).

\section{Assays \\ Western blot. Subconfluent monolayers of MB49 cells were treated with $2 \mathrm{mg} / \mathrm{ml} \mathrm{BCG}$ with or without $10 \mu \mathrm{M}$ 15-d-PGJ2 (Cayman Chemical, Ann Arbor, Michigan) for 24 hours for total lysates and for 45 minutes for nuclear extracts. NE-PER ${ }^{\mathrm{TM}}$ Nuclear and Cytoplasmic Extraction Reagents were used to obtain the nuclear compartment. Western blots were performed, as described previously. ${ }^{9}$ PPARg antibody (sc-H-100, Santa Cruz Biotechnology, Santa Cruz, California) was used. To evaluate the loading control, $\beta$-actin (A5441, Sigma®) or histone H1 (FL-219, Santa Cruz Biotechnology) antibody was used on stripped membranes.}

Gene reporter. To study PPARg transcriptional activity MB49 and NIH-3T3 cells were transiently transfected with $20 \mu \mathrm{g}$ of a PPRE-luciferase reporter plasmid. MAC activation was evaluated by $\mathrm{NF}-\kappa \mathrm{B}$ activation using transient transfection with $20 \mu \mathrm{g}$ of an NF- $\kappa \mathrm{B}-$ luciferase reporter plasmid in RAWs, as previously described. ${ }^{20}$ Luciferase activity was determined using the Dual-Luciferase® Reporter
Assay System in a Triathler luminometer liquid scintillation counter (Hidex, Turku, Finland) and normalized to constitutive Renilla activity.

Cell viability. Subconfluent monolayers of NIH-3T3 cells growing in 24-well plates were treated with BCG $(2 \mathrm{mg} / \mathrm{ml})$, 15-d-PGJ2 $(10 \mu \mathrm{M}), \mathrm{RO}(10 \mu \mathrm{M})$ or BADGE $(10 \mu \mathrm{M})$ for 48 hours. Cell viability was determined by cell counting with trypan blue.

\section{In Vivo Tumor Growth, Peritoneal MAC Extraction and NO Production}

Eight-week-old C57BL/6J male mice were subcutaneously injected with $5 \times 10^{5} \mathrm{MB} 49$ cells. Animals were treated with intratumor BCG $(2 \mathrm{mg} / \mathrm{ml})$ with or without BADGE $(100 \mu \mathrm{M} / 100 \mu \mathrm{l}$ per mouse) twice per week or RO $(8 \mathrm{mg} / \mathrm{kg}$ per day) in drinking water for 30 days. Control mice were equally injected with saline solution. Tumor size was measured twice per week and calculated using the equation, $(\mathrm{D} \times \mathrm{d})^{1 / 2}$, where $\mathrm{D}$ represents the larger diameter and $d$ represents the smaller diameter in $\mathrm{mm}$. At 30 days MACs were obtained by washing the peritoneal cavity with cool phosphate buffered saline-ethylenediaminetetraacetic acid $(0.02 \%)$. MACs were purified from peritoneal washes by plastic adhesion for 2 hours.

NO was determined in 24-hour MAC culture supernatants from tumor bearing mice by the Griess reaction, as previously described. ${ }^{8}$

\section{Activity}

Phagocytes. Phagocytic activity was determined as the cfu and by Ziehl-Neelsen staining. To this end, $3 \times 10^{5}$ RAWs/ml were treated for 24 hours with RO $(10 \mu \mathrm{M})$ and incubated for 1 hour with BCG ( $1 \mathrm{mg} / \mathrm{ml})$. After washing with phosphate buffered saline, cells were lysed with $5 \%$ saponin and seeded on Löwenstein-Jensen solid medium. Colonies were quantified after 30 days. Phagocytic activity is reported in $\mathrm{cfu} / \mathrm{ml}$.

Ziehl-Neelsen staining was used to evaluate the number of bacilli in MACs and RAWs incubated with BCG for 2 hours, as seen as a pink area in cells. Treated cells were fixed with methanol for 20 minutes, incubated in $100 \%$ fuchsin for 1 hour and washed with bleach $(3 \% \mathrm{HCl}$ in $95 \%$ ethyl alcohol) until discoloration. Glasses were incubated with methylene blue for 20 minutes.

Matrix metalloproteinase. MMP-9 was determined as a $105 \mathrm{kDa}$ collagenolytic band by zymography in culture supernatants from peritoneal MACs deprived of serum for 24 hours, as previously described. ${ }^{21}$ It is expressed in $\mathrm{AU} / 10^{4}$ cells.

\section{Immunohistochemistry}

$\alpha$-SMA expression was determined and Masson trichrome staining was performed in paraffin embedded sections of subcutaneous MB49 tumors from mice treated with BCG with or without RO, as described previously. ${ }^{9}$

\section{Ethics Statement}

Mice from our Institute Animal Care Division were handled in accordance with the international procedure for Care and Use of Laboratory Animals. Protocols were approved by the Institutional Review Board CICUAL, Fac- 
ultad de Medicina, Universidad de Buenos Aires, Argentina.

\section{Statistical Analysis}

Three independent experiments were performed and only one is shown. Results are expressed as the mean $\pm \mathrm{SD}$ of 6 replicates per group. Statistically significant values were compared using ANOVA and the Bonferroni contrast using the InStat@ 3.01 with $\mathrm{p}<0.05$ considered statistically significant.

\section{RESULTS}

\section{BCG Induced PPARg and}

\section{Transcriptional Activity in MB49 Cell Line}

BCG induced PPARg expression in the BC cell line MB49 upon 24 hours of treatment (fig. 1, A). Under basal conditions PPARg was absent in nuclear extracts, while it was translocated to the nucleus after BCG or 15-d-PGJ2 alone or combined with BCG treatment (fig. 1, B). A reporter gene assay with PPRE showed that BCG and 15-d-PGJ2 increased PPARg transcriptional activity, while combined treatment of BCG plus 15-d-PGJ2 had a greater effect than each of them separately (fig. 1, C). Thus, the PPARg ligand increased the PPARg transcriptional activity induced by BCG in MB49 cells.

\section{In Vivo RO}

Reversed tumor growth inhibition induced by BCG. We evaluated the effect of BCG and RO on in vivo MB49 tumor growth. Results revealed that $R O$ did not affect tumor development compared to control mice, while late in tumor progression it partially reversed the inhibition caused by BCG administration (fig. 2, A). The same effect was recorded when we analyzed the effect of the PPARg antagonist BADGE (fig. 2, B). BADGE alone did not affect tumor progression and it partially reversed the inhi- bition caused by BCG treatment. The 2 experiments indicated that use of a PPARg agonist or antagonist partly affected the mechanisms of BCG antitumor activity.

Inhibited BCG activated MACs. Tumor bearing mice treated with BCG had an almost threefold higher number of MACs than control mice. Enhancement of the number of MACs by BCG was associated with increased metabolic activity and NO production. The number, metabolic activity and NO production of MACs increased by BCG were partially inhibited by BCG plus RO. Phagocytic activity was not modified by BCG, while RO with or without BCG inhibited this activity. MMP-9 activity of MACs from BCG plus RO tumor bearing mice was decreased compared to that in control mice (see table). Therefore, in vivo activation of MACs was inhibited by RO.

MAC activation by BCG was also studied in RAWs. As expected, BCG induced NF- $\kappa$ B transcriptional activity, which was completely inhibited upon combined treatment with RO (fig. 3, A). As evaluated by BCG according to cfu, RAW phagocytic activity was significantly inhibited by RO (fig. 3, B). A similar result was observed for the Ziehl-Neelsen staining assay (fig. 3, C), on which fewer bacilli were detected in RAWs treated with RO.

\section{PPARg Ligands Inhibited Fibroblast Activation Induced by BCG}

We then analyzed fibroblast proliferation in the presence of BCG with 15-d-PGJ2 or RO (fig. 4, A). Fibroblast proliferation induced by BCG was significantly inhibited by 15-d-PGJ2 and RO. The effect of the 2 PPARg agonists was reversed in the presence of BADGE, indicating that the inhibition of fibroblast proliferation depended on ligand PPARg activation. To evaluate PPARg transcriptional activity
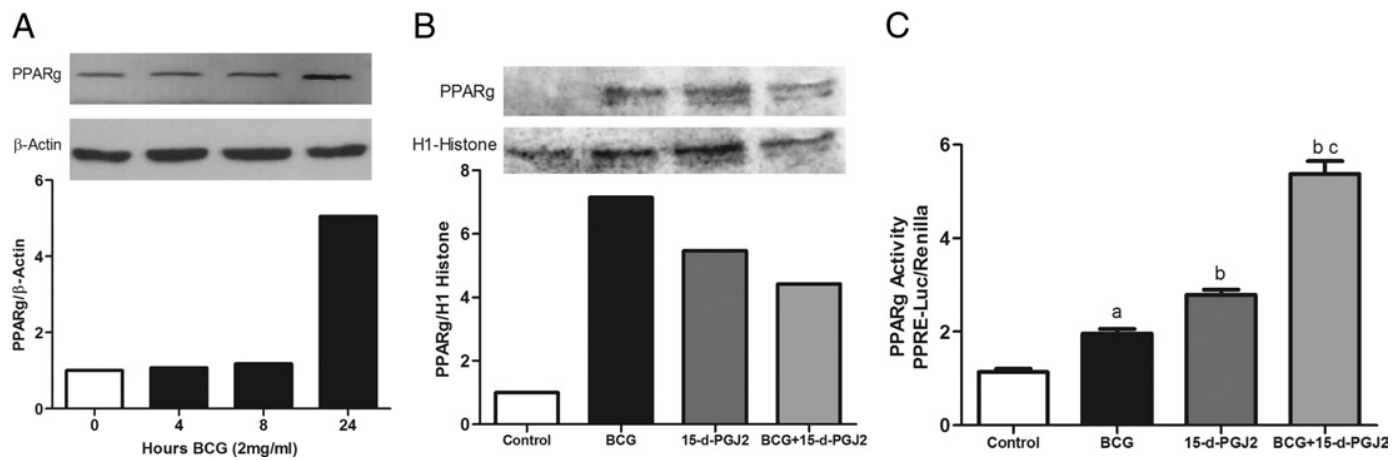

Figure 1. A, PPARg expression with time in MB49 cells under BCG treatment. $\beta$-actin expression was used to assess PPARg relative expression. BCG increased PPARg expression fivefold over control level after 24-hour treatment. $B$, nuclear PPARg was detected 45 minutes after BCG, 15-d-PGJ2 or BCG plus 15-d-PGJ2 treatment. Increased PPARg expression was 7.5, 5.5 and 3.5-fold greater, respectively, than in controls. C, PPARg transcriptional activity in MB49 subconfluent monolayers transfected with PPRE-luciferase plasmid and Renilla plasmid after 24 hours of different treatments. Activity is shown as luciferase counts per second compared to Renilla activity and referred to control. $a, \mathrm{p}<0.001$ vs control. $b, \mathrm{p}<0.0001$ vs control. $c, \mathrm{p}<0.0001$ vs BCG or $15-\mathrm{d}-\mathrm{PGJ}$. 
A

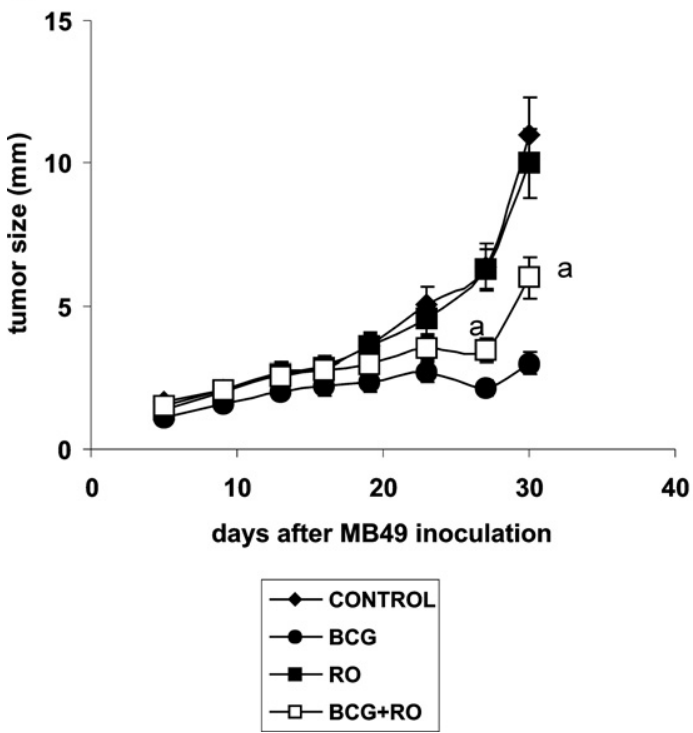

B

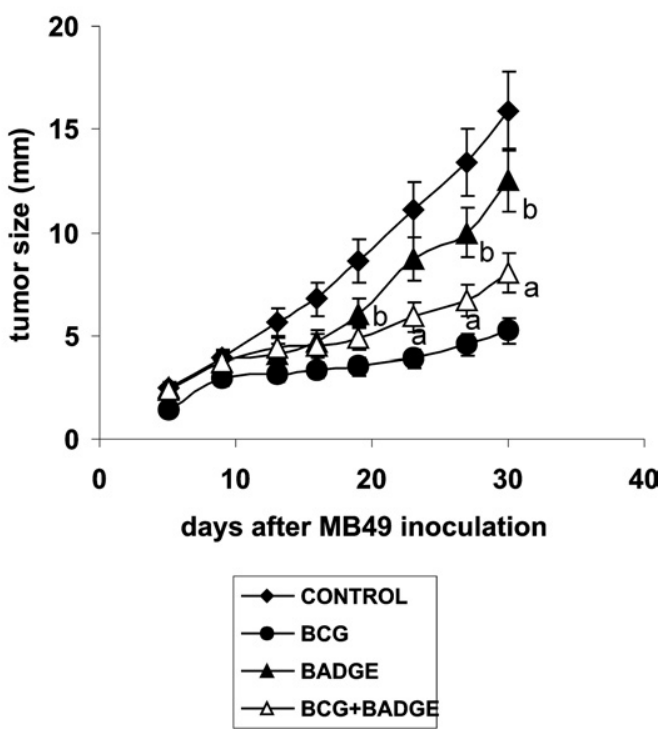

Figure 2. Subcutaneous MB49 tumor growth in mice. $A$, treatment with BCG, RO or BCG plus RO. $a, \mathrm{p}<0.01$ vs control and $B C G$. $B$, treatment with BCG, BADGE or BCG plus BADGE, as described. $a, \mathrm{p}<0.05$ vs BCG. $b, \mathrm{p}<0.05$ vs control.

in fibroblasts, we performed a reporter gene assay. BCG increased PPARg transcriptional activity, which was blocked by 15-d-PGJ2. This inhibition was reversed in the presence of BADGE (fig. 4, B).

\section{BCG Induced and RO Inhibited Collagen and $\alpha$-SMA in MB49 Tumors}

Intratumor inoculation of BCG induced an important collagen deposit, seen as blue fibers using Masson trichrome stain. This collagen deposit was lower in tumors from mice treated with BCG plus RO (fig. 5, A). Immunohistochemistry revealed that BCG induced $\alpha$-SMA expression, while combined treatment with RO decreased it (fig. 5, $B$ ). This suggests that the number of activated fibroblasts was lower in tumors from mice that received BCG plus RO therapy.

\section{DISCUSSION}

Intravesical BCG therapy is the best treatment to date to prevent NMI BC progression and recur- rence. ${ }^{5,15}$ The exact mechanism of action of BCG is not fully known but it seems to evoke direct effects on tumor cells and indirect effects mediated by the immune system and stromal cells..$^{5,6,9}$

PPARs are involved in the regulation of various pathological processes and their activation causes tumor growth inhibition or tumor cell differentiation. ${ }^{13,14}$ We previously noted that BCG induced PPARg expression in human and murine BC cells in vitro as well as in MB49 tumors. ${ }^{18}$ In vitro growth inhibition by BCG was enhanced by 15-d-PGJ2 and reverted by BADGE. This suggested that BCG has the ability to induce functional PPARg, which can respond to the endogenous ligand. In the current study BCG induced not only the expression but also the translocation to the nucleus and transcriptional activity of PPARg in BC MB49 cells. This activation was also increased by 15-d-PGJ2. The nuclear translocation of NF- $\kappa \mathrm{B}$ and degradation of IkB- $\alpha$ induced by BCG were partially attenuated by 15 -d-PGJ2

Activation of MACs from tumor bearing mice was inhibited by $R O$

\begin{tabular}{|c|c|c|c|c|}
\hline & Mean \pm SD Control & Mean \pm SD RO & Mean \pm SD BCG & Mean $\pm \mathrm{SD} B C G+\mathrm{RO}^{*}$ \\
\hline \multicolumn{5}{|l|}{ MACs: } \\
\hline No. $\left(10^{6} /\right.$ mouse $)$ & $1.1 \pm 0.2$ & $1.0 \pm 0.2$ & $2.8 \pm 0.3 \dagger$ & $1.5 \pm 0.2$ \\
\hline Metabolic activity (MTS 492 nm) & $0.22 \pm 0.02$ & $0.28 \pm 0.02$ & $0.49 \pm 0.05 \dagger$ & $0.30 \pm 0.03$ \\
\hline $\mathrm{NO} /$ nitrite (mol/10 6 cells) & $5.00 \pm 0.04$ & $5.00 \pm 0.05$ & $31.00 \pm 0.03 \dagger$ & $17.60 \pm 0.1$ \\
\hline Phagocytic index (BCG/MAC) $\ddagger$ & $12 \pm 3$ & $3 \pm 3 \S$ & $11 \pm 3$ & $4 \pm 2$ \\
\hline MMP-9 activity (AU/10 6 cells) & $5.00 \pm 2$ & $4.40 \pm 2$ & $7.75 \pm 2$ & $3.35 \pm 1$ \\
\hline
\end{tabular}

* Bonferroni contrast and ANOVA $p<0.05$ vs BCG.

$\dagger$ Bonferroni contrast and ANOVA $p<0.01$ vs control.

$\ddagger$ Assessed by counting number of bacilli in MACs after Ziehl-Neelsen staining.

$\S$ Bonferroni contrast and ANOVA $p<0.05$ vs control. 

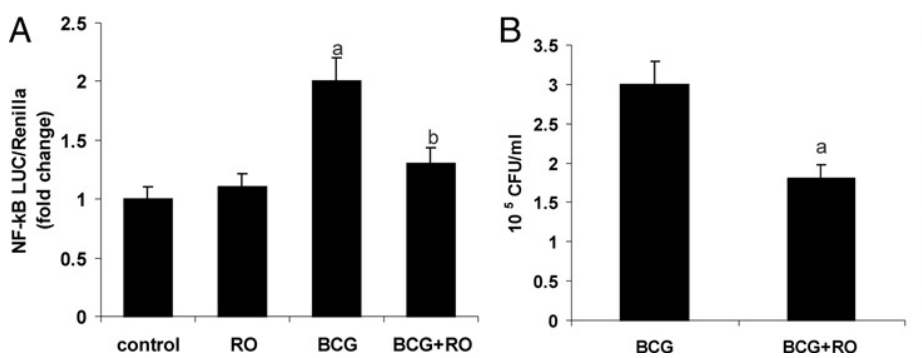

C

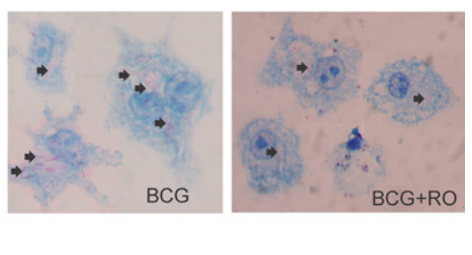

Figure 3. A, subconfluent RAW monolayers transfected with plasmid containing element that responds to NF- $\kappa \mathrm{B}-$ luciferase and Renilla plasmid. Cells were treated or not treated with BCG $(1 \mathrm{mg} / \mathrm{ml})$ and RO $(10 \mu \mathrm{M})$ for 24 hours. Activity is shown as luciferase-to-Renilla cps and referred to control. $a, \mathrm{p}<0.001$ vs untreated. $b, \mathrm{p}<0.01$ vs BCG. $B$, RAW phagocytic capacity reported in cfu ml. $a$, Student $\mathrm{t}$ test $\mathrm{p}<0.0208$. $C$, phagocytic activity. Arrow indicates BCG inside RAWs. Ziehl-Neelsen stain, reduced from $\times 1,000$.

(data not shown). Thus, BCG induced the direct inhibition of $\mathrm{BC}$ cells, in part by PPARg activation and NF- $\kappa$ B inactivation.

Paradoxically, in vivo we also noted that the synthetic agonist RO reversed the inhibitory growth activity induced by BCG. However, the inhibition of tumor growth induced by BCG also depended on PPARg activity since it was inhibited by BADGE. Thus, PPARg was intrinsically involved in BCG antitumor activity. We speculate that the agonist RO can affect other mechanisms involved in BCG immunotherapy, such as the host immune response and tissue remodeling. PPARg agonists can abolish immune activation. ${ }^{22-24}$ However, to our knowledge there is no information on the role of PPARg activation in tissue remodeling in response to BCG. To explain this effect, we focused on 2 key elements of tumor stroma involved in the BCG mechanism of action, including MACs and fibroblasts.
Recent results showed that BCG not only targets immune and $\mathrm{BC}$ cells but also induces the activation of fibroblasts directly or indirectly through MAC activation. ${ }^{9}$ Since MACs have a central role in the regulation of the immune response as well as in tissue remodeling, we evaluated some MAC parameters and their modulation by PPARg agonists. In tumor bearing animals BCG treatment increased the number, metabolic activity and NO production of peritoneal MACs, while phagocytic capacity and MMP-9 activity were not modified. Co-administration of BCG plus RO partially inhibited the number, metabolic activity and NO production of MACs. RO alone or combined with BCG decreased MAC phagocytic capacity, while BCG plus RO decreased the activity of MMP-9 activity (which was used for its migration) compared to controls. In vitro using MAC RAWs we confirmed that RO inhibited NF- $\mathrm{B}$ activation and, thus, decreased phagocytic capacity.
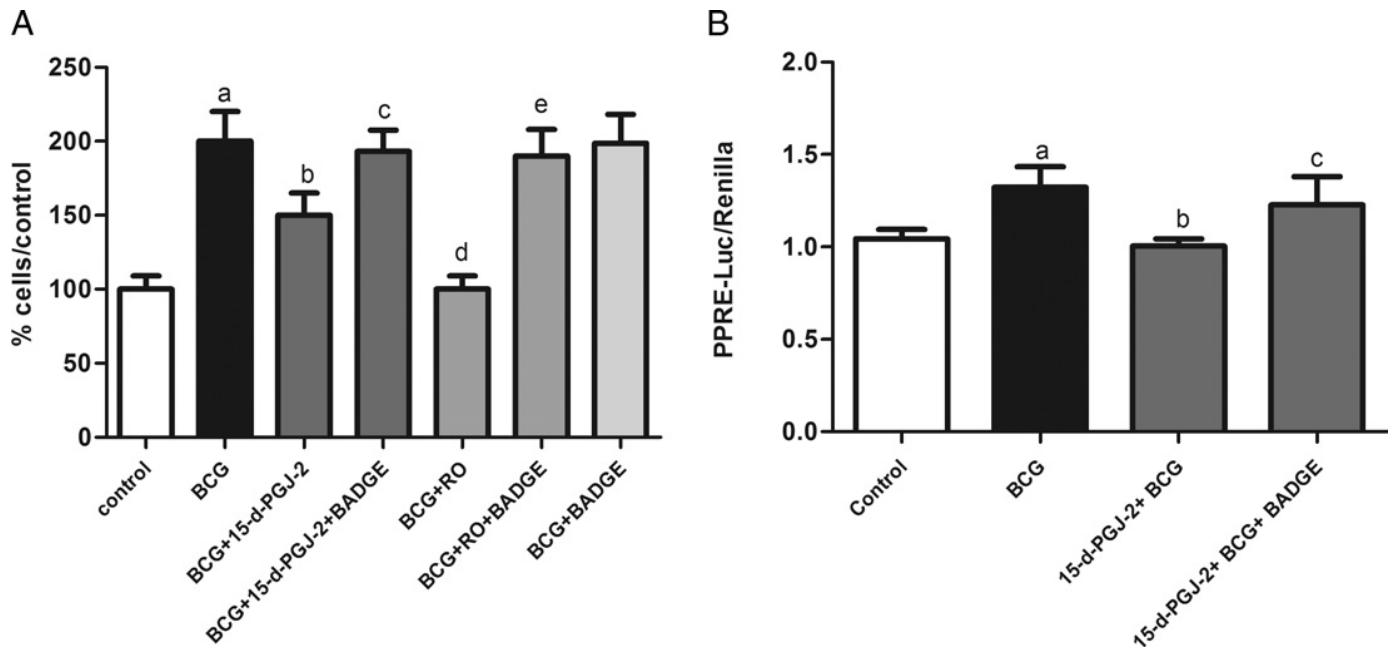

Figure 4. $A$, number of NIH-3T3 fibroblasts after 48 hours of different treatments. $a, \mathrm{p}<0.001$ vs control. $b, \mathrm{p}<0.05$ vs $\mathrm{BCG}$. $c, \mathrm{p}<0.05$ vs 15-d-PGJ2 plus BCG. $d, \mathrm{p}<0.001$ vs BCG. $e, p<0.001$ vs RO plus BCG. B, PPARg transcriptional activity in NIH-3T3 subconfluent monolayers transfected with PPRE-luciferase and Renilla plasmids after 24-hour treatment with BCG, 15-d-PGJ2 and BADGE. Activity is expressed as luciferase cps vs Renilla activity and referred to control. $a, \mathrm{p}<0.01$ vs control. $b, \mathrm{p}<0.01$ vs BCG. $c, \mathrm{p}<0.01$ vs 15-d-PGJ2 plus BCG. 


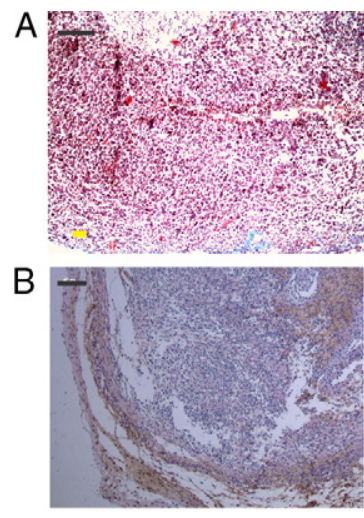

control

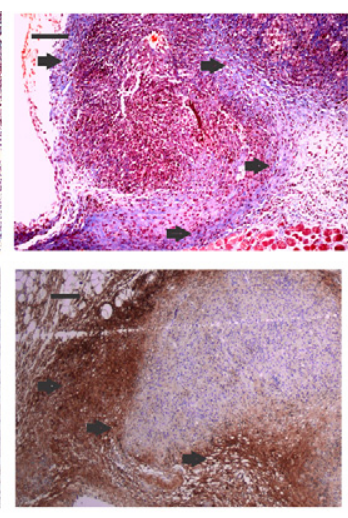

BCG

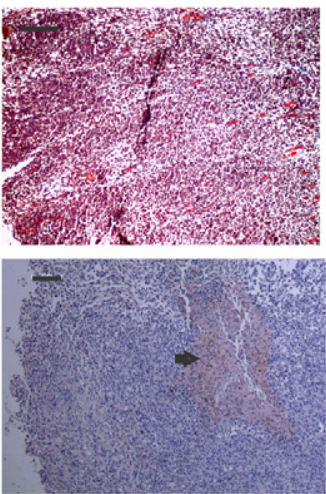

RO

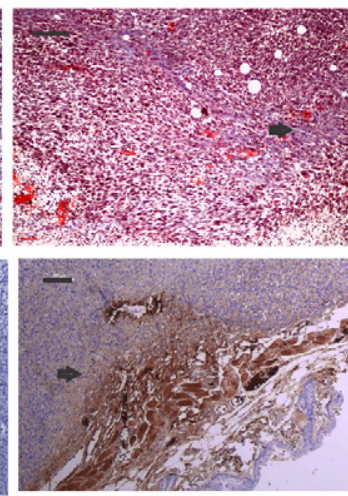

RO BCG

Figure 5. Histological sections of subcutaneous MB49 tumors from mice treated with BCG, RO or RO plus BCG. $A$, note collagen fibers (arrows, light blue areas). Masson trichrome stain, scale bar represents $100 \mu \mathrm{m}$. $B$, immunohistochemistry for $\alpha$-SMA. Black arrow indicates positive expression. Scale bar represents $100 \mu \mathrm{m}$.

These results led us to hypothesize that RO partly inhibits the BCG antitumor response by inhibiting MAC function.

Fibroblast activation was also involved in BCG antitumor therapy. Previously, we observed that BCG inhibits tumor growth by inducing the deposition of collagen fibers on surrounding tumor cells as well as $\alpha$-SMA and fibroblast growth factor-2 expression. ${ }^{8,9}$ In this study the in vitro fibroblast proliferation induced by BCG was inhibited by PPARg agonists. This was also evident in vivo, in which decreased fibroblast activation and differentiation were detected by a decrease in collagen deposition and $\alpha$-SMA expression in tumors from mice treated with BCG plus RO. Also, we detected that NIH-3T3 fibroblast treatment with BCG plus RO enhanced collagenolytic MMP-2 activity (data not shown). The inhibition of collagen and the increase in MMP-2 in fibroblasts may explain the decreased collagen in BCG plus RO treated MB49 tumors.

The antifibrotic activity of thiazolidinediones was described in other experimental models, in which thiazolidinediones inhibited collagen synthesis in liver and lung fibrosis. ${ }^{25,26}$ Inhibition of the migration, proliferation and differentiation by $\mathrm{RO}$ was noted in cultures of human lung fibroblasts and pioglitazone inhibited matrix synthesis in dermal fibroblasts. ${ }^{27,28}$ Taken together, our results and those of others suggest that these PPARg agonists are involved in the inhibition of some steps of tissue remodeling. Particularly, RO treatment inhibits the function not only of MACs but also of fibroblasts, reversing part of the BCG response.

Since we tested only the effect of RO, we cannot extrapolate our results to any other PPARg agonist. However, this could be extended to other thiazolidinediones because they have a similar mechanism of action for PPARg activation. Recently, a more potent class of PPARg agonists was developed from a series of 1,1-bis(3V-indolyl)-1-(p-substituted phenyl) methanes. These compounds show significant antitumor activity and they are significantly more potent inhibitors of BC growth than RO. ${ }^{25,29}$ Since the mechanism of action of this class of PPARg agonists differs from that of thiazolidinediones, they could be useful in combination with BCG. Thus, it is important to perform further research in this area.

Notably, the subcutaneous implantation model has some limitations compared with the clinical setting. For example, BCG persistence at the injection site in the extracellular compartment is distinctly different than intravesical administration. In the bladder normal urothelial cells as well as tumor cells and MACs can engulf BCG. This is an important step in the antitumor response. However, with subcutaneous inoculation normal urothelial cells are absent. Even with these limitations, this model is a valid approach for studying tumor cells and MACs.

\section{CONCLUSIONS}

Results show that BCG exerts its antitumor activity in part by mediation by PPARg induction and activation in $\mathrm{BC}$ cells. It could be hypothesized that endogenous PPARg agonist levels are involved in this tumor cell death. However, exogenous PPARg agonists such as RO would not be a favorable therapeutic modality for $\mathrm{BC}$ because they can inhibit the tissue remodeling needed for an overall satisfactory BCG response.

\section{ACKNOWLEDGMENTS}

Dr. Claudia Arguelles, Instituto Producción de Biológicos, provided BCG. Lic Rut Slimovich, Instituto 
Nacional de Producción de Biológicos, provided technical assistance. Lic Maria Adela Jasnis critically reviewed the manuscript. Mycobacterium tuberculo- sis (Pasteur 1172 P2 strain) was provided by the Instituto Nacional de Producción de Biológicos, Buenos Aires, Argentina.

\section{REFERENCES}

1. Greenlee RT, Hill-Harmon MB, Murray T et al: Cancer statistics, 2001. CA Cancer J Clin 2001: 51: 15

2. de Braud F, Maffezzini M, Vitale V et al: Bladder cancer. Crit Rev Oncol Hematol 2002; 41: 89.

3. Jemal A, Siegel R, Ward E et al: Cancer statistics, 2009. CA Cancer J Clin 2009; 59: 225.

4. Sauter G, Algaba F, Amin MB et al: Tumours of the urinary system. In: World Health Organization Classification of Tumours Pathology and Genetics: Tumours of the Urinary System and Male Genital Organs. Edited by JI Epstein, JN Eble, I Sesterhenn et al. Lyon: IARC Press 2004; pp 89-157.

5. Riemensberger J, Bohle A and Brandau S: IFNgamma and IL-12 but not IL-10 are required for local tumour surveillance in a syngeneic model of orthotopic bladder cancer. Clin Exp Immunol 2002: 127: 20.

6. Suttmann $\mathrm{H}$, Riemensberger J, Bentien $\mathrm{G}$ et al: Neutrophil granulocytes are required for effective bacillus Calmette-Guerin immunotherapy of bladder cancer and orchestrate local immune responses. Cancer Res 2006; 66: 8250.

7. Sandes E, Lodillinsky C, Cwirenbaum R et al: Cathepsin B is involved in the apoptosis intrinsic pathway induced by bacillus Calmette-Guerin in transitional cancer cell lines. Int $\mathrm{J}$ Mol Med 2007: 20: 823.

8. Alvarez V, Lodillinsky C, Umerez S et al: Inhibition of bacillus Calmette-Guerin-induced nitric oxide in bladder tumor cells may improve BCG treatment. Int J Mol Med 2005; 16: 565.

9. Lodillinsky C, Langle Y, Guionet A et al: Bacillus Calmette Guerin induces fibroblast activation both directly and through macrophages in a mouse bladder cancer model. PLoS One 2010; 5: e13571.

10. Lemberger T, Desvergne B and Wahli W: Peroxisome proliferator-activated receptors: a nuclear receptor signaling pathway in lipid physiology. Annu Rev Cell Dev Biol 1996; 12: 335.
11. Forman BM, Tontonoz P, Chen J et al: 15-Deoxydelta 12,14-prostaglandin $\mathrm{J} 2$ is a ligand for the adipocyte determination factor PPAR gamma. Cell 1995; 83: 803

12. Wang T, Xu J, Yu X et al: Peroxisome proliferatoractivated receptor gamma in malignant diseases. Crit Rev Oncol Hematol 2006; 58: 1.

13. Yoshida $K$, Hirose $Y$, Tanaka $T$ et al: Inhibitory effects of troglitazone, a peroxisome proliferatoractivated receptor gamma ligand, in rat tongue carcinogenesis initiated with 4-nitroquinoline 1-oxide. Cancer Sci 2003; 94: 365.

14. Matsuyama $M$ and Yoshimura R: A novel approach to anticancer therapies: peroxisome proliferator activator-receptor-gamma as a new target therapy in the treatment of human urological cancer. Endocr Metab Immune Disord Drug Targets 2009; 9: 76.

15. Plissonnier ML, Fauconnet $\mathrm{S}$, Bittard $\mathrm{H}$ et al: Insights on distinct pathways of thiazolidinediones (PPARgamma ligand)-promoted apoptosis in TRAll-sensitive or -resistant malignant urothelial cells. Int J Cancer 2010; 127: 1769.

16. Nakashiro Kl, Hayashi Y, Kita A et al: Role of peroxisome proliferator-activated receptor gamma and its ligands in non-neoplastic and neoplastic human urothelial cells. Am J Pathol 2001; 159: 591.

17. Possati $L$, Rocchetti $R$, Talevi $S$ et al: The role of peroxisome proliferator-activated receptor gamma in bladder cancer in relation to angiogenesis and progression. Gen Pharmacol 2000; 35: 269

18. Lodillinsky C, Umerez MS, Jasnis MA et al: Bacillus Calmette-Guerin induces the expression of peroxisome proliferator-activated receptor gamma in bladder cancer cells. Int J Mol Med 2006; 17: 269

19. Inamoto T, Azuma H, Ubai T et al: Association of PPAR- gamma with BCG immunotherapy. Med Hypotheses 2009; 72: 231.

20. Diaz Bessone MI, Berardi DE, Campodonico PB et al: Involvement of PKC delta (PKCdelta) in the resistance against different doxorubicin analogs. Breast Cancer Res Treat 2011; 126: 577.
21. Urtreger AJ, Grossoni VC, Falbo KB et al: Atypical protein kinase C-zeta modulates clonogenicity, motility, and secretion of proteolytic enzymes in murine mammary cells. Mol Carcinog 2005; 42: 29.

22. Dworzanski T, Celinski K, Korolczuk A et al: Influence of the peroxisome proliferator-activated receptor gamma (PPAR-gamma) agonist, rosiglitazone and antagonist, biphenol-A-diglicydyl ether (BADGE) on the course of inflammation in the experimental model of colitis in rats. J Physiol Pharmacol 2010; 61: 683.

23. Jung K, Tanaka A, Fujita $H$ et al: Peroxisome proliferator-activated receptor gamma-mediated suppression of dendritic cell function prevents the onset of atopic dermatitis in NC/Tnd mice. J Allergy Clin Immunol 2011; 127: 420.

24. Rampino T, Ranghino A, Guidetti $C$ et al: Activation of PPARgamma enhances in vitro the immunosuppressive effect of cyclosporine on T lymphocytes. Transpl Immunol 2007; 18: 32.

25. Wang G, Qi B, Zheng $\mathrm{H}$ et al: (Z)-5-(4-Methoxybenzylidene)thiazolidine-2,4-dione, a novel readily available and orally active glitazone, attenuates the bleomycin-induced pulmonary fibrosis in vivo. Biol Pharm Bull 2011; 34: 219.

26. Wang XM and Chen DF: Effects of rosiglitazone on Kruppul-like factor 6 (KLF6) signaling in the livers of rats with nonalcoholic fatty liver fibrosis Zhonghua Gan Zang Bing Za Zhi 2007; 15: 649.

27. Lin Q, Fang LP, Zhou WW et al: Rosiglitazone inhibits migration, proliferation, and phenotypic differentiation in cultured human lung fibroblasts. Exp Lung Res 2010; 36: 120.

28. Zhang GY, Cheng T, Zheng MH et al: Peroxisome proliferator-activated receptor-gamma (PPARgamma) agonist inhibits transforming growth factor-beta1 and matrix production in human derma fibroblasts. J Plast Reconstr Aesthet Surg 2010; 63: 1209 .

29. Kassouf W, Chintharlapalli S, Abdelrahim M et al: Inhibition of bladder tumor growth by 1,1bis(3'-indolyl)-1-(p-substitutedphenyl)methanes: a new class of peroxisome proliferator-activated receptor gamma agonists. Cancer Res 2006; 66: 412. 